\title{
UNIVERSAL CONVEXITY AND UNIVERSAL STARLIKENESS OF POLYLOGARITHMS
}

\author{
ANDREW BAKAN, STEPHAN RUSCHEWEYH, AND LUIS SALINAS
}

(Communicated by Walter Van Assche)

\begin{abstract}
A deep result of J. Lewis (1983) shows that the polylogarithms $L i_{\alpha}(z):=\sum_{k=1}^{\infty} z^{k} / k^{\alpha}$ map the open unit disk $\mathbb{D}$ centered at the origin oneto-one onto convex domains for all $\alpha \geq 0$. In the present paper this result is generalized to the so-called universal convexity and universal starlikeness (with respect to the origin) in the slit-domain $\Lambda:=\mathbb{C} \backslash[1, \infty)$, introduced by S. Ruscheweyh, L. Salinas and T. Sugawa (2009). This settles a conjecture made in that work and proves, in particular, that $L i_{\alpha}(z)$ maps an arbitrary open disk or half-plane in $\Lambda$ one-to-one onto a convex domain for every $\alpha \geq 1$.
\end{abstract}

\section{INTRODUCTION}

For a domain $\Omega$ in the complex plane $\mathbb{C}$ let $\operatorname{Hol}(\Omega)$ be the set of all holomorphic functions in $\Omega$. Denote by $\mathbb{D}$ the open unit disk $\{z \in \mathbb{C}:|z|<1\}$ and by $\Lambda$ the slit-domain $\mathbb{C} \backslash[1, \infty)$ in $\mathbb{C}$.

Our work deals with the so-called polylogarithms (see [5, 6])

$$
L i_{\alpha}(z):=\sum_{k=1}^{\infty} \frac{z^{k}}{k^{\alpha}}, z \in \mathbb{D}, \alpha \in \mathbb{R},
$$

which are analytic in $\Lambda$ as it follows from the well-known integral representation [4, (3), p.27]

$$
\frac{L i_{\alpha}(z)}{z}=\frac{1}{\Gamma(\alpha)} \int_{0}^{1} \frac{\log ^{\alpha-1}(1 / t)}{1-t z} d t, \quad z \in \Lambda, \alpha>0,
$$

the formulas $L i_{\alpha-n}(z)=(z d / d z)^{n} L i_{\alpha}(z), n=1,2, \ldots$, and a suitable application of analytic continuation from $\mathbb{D}$ into $\Lambda$.

J. Lewis, in his remarkable paper [10, studied the restrictions of $L i_{\alpha}$ to the unit disk $\mathbb{D} \subset \Lambda$ and showed that for all $\alpha \geq 0$ they map $\mathbb{D}$ one-to-one onto convex domains and this is not true for every $\alpha<0$. Note that, by the classical Alexander theorem [3. Theorem 2.12, p.43], this implies that for all $\alpha \geq-1$ the functions $L i_{\alpha}=z L i_{\alpha+1}^{\prime}$ map $\mathbb{D}$ one-to-one onto a domain which is starlike with respect to the origin [3, p.40] but for every $\alpha<-1$ this property is not valid.

Received by the editors December 19, 2012 and, in revised form, May 10, 2013.

2010 Mathematics Subject Classification. Primary 30C45, 30H10; Secondary 44A15.

Key words and phrases. Convex functions, polylogarithms, universally convex functions, universally starlike functions, Pick functions.

The second and third authors acknowledge support from FONDECYT, Grant 1100805, from Basal Project FB0821 CCTVal-Centro Científico Tecnológico de Valparaíso, and from Anillo Project ACT119. This work was completed while the first author was visiting Würzburg University, supported by the German Academic Exchange Service (DAAD, grant 322-A/11/05274). 
In 14, Def.1.3, 1.4, pp.290-291] the notions of universal starlikeness and universal convexity have been introduced for functions in $\operatorname{Hol}(\Lambda)$ :

Definition 1. A function $f \in \operatorname{Hol}(\Lambda)$, with $f(0)=0$ and $f^{\prime}(0)=1$, is called universally starlike if it maps every circular domain $\Omega \subset \Lambda$ with $0 \in \Omega$ one-to-one onto a domain starlike with respect to the origin. It is called universally convex if it maps every circular domain $\Omega \subset \Lambda$ one-to-one onto a convex domain.

Note that in this definition circular domains are meant to be open disks or open half-planes in $\mathbb{C}$.

Actually, in 14, the study of universally convex and starlike functions has been embedded into a theory of universally prestarlike functions of order $\gamma<1$, and it has been proved in [14, Theorems 1.5, 1.6, p.291] that a function $f$ is universally starlike (with respect to the origin) or universally convex iff $f$ is universally prestarlike of order $1 / 2$ or of order 0 , respectively. In [14, Corollary 1.1, p.289] an explicit analytic representation for universally prestarlike functions of order $\gamma<1$ has been established. The following theorem is a special case.

Theorem A ([14, Corollary 1, p.289]). Let $f \in \operatorname{Hol}(\Lambda)$, with $f(0)=0, f^{\prime}(0)=1$. Then $f$ is universally starlike respectively universally convex if and only if there exists a probability measure $\mu$ on $[0,1]$ such that

$$
\frac{f(z)}{z}=e^{\int[0,1]} \log \frac{1}{1-t z} d \mu(t) \quad, \quad z \in \Lambda,
$$

respectively

$$
f^{\prime}(z)=e^{2 \cdot \int_{[0,1]} \log \frac{1}{1-t z} d \mu(t)}, \quad z \in \Lambda .
$$

Recall the definition of the Hadamard product ' $*$ ' (convolution) of two functions $g(z)=\sum_{k=0}^{\infty} g_{k} z^{k}, h(z)=\sum_{k=0}^{\infty} h_{k} z^{k} \in \operatorname{Hol}(\mathbb{D}),(g * h)(z):=\sum_{k=0}^{\infty} g_{k} h_{k} z^{k}$. The following result describes an important feature of universally convex and starlike functions (again a special case of the classes of universally prestarlike functions of order $\gamma \leq 1)$.

Theorem B ([13, Corollary 1.2]). The sets of universally convex respectively universally starlike functions are closed under convolution (Hadamard product).

For the universally convex case this is a direct analogue to the Pólya-Schoenberg theorem for convex univalent functions in $\mathbb{D}$ (see [12]). There is no analogue in $\mathbb{D}$ for the universally starlike case of Theorem B.

In the spirit of Lewis' theorem mentioned above the following result has been obtained in [14, Theorem 1.7, p.292]. Note that the classical form of Alexander's theorem is not valid in the 'universal' setting. Therefore, passing between universal convexity and universal starlikeness is slightly different from the classical unit disk case.

Theorem C ([14, Theorem 1.7]). The functions $L i_{\alpha}$ are universally starlike for $\alpha=0$ and $\alpha \geq 1$. They are universally convex for $\alpha=0, \alpha=1$ and $\alpha \geq 2$. For $\alpha \in(0,1)$ they are not universally convex.

In [14 the following extension of Theorem Chas been conjectured. 
Conjecture 1 ([14, p. 292]). The functions $L i_{\alpha}$ are universally starlike for $\alpha \geq$ 0 . They are universally convex for $\alpha \geq 1$ (and for $\alpha=0$, as it follows from Theorem C].

It is our aim to verify this conjecture, actually without referring to Theorem C. We first show that the truth of a small special case of this claim is enough to settle it completely.

Lemma 1. Assume there exists an $\varepsilon>0$ such that $L i_{\alpha}$ is universally starlike for all $0<\alpha<\varepsilon$. Then Conjecture 1 is valid.

Proof. Universal starlikeness and universal convexity of $L i_{0}$ are obvious because (1.2) and (1.3) are valid for $f(z)=L i_{0}(z)=z /(1-z)$ and $\mu([0, x])=\chi_{\{1\}}(x)$, $x \in[0,1]$, where $\chi_{A}(x)$ denotes the characteristic function of $A \subset \mathbb{R}$. Let $\alpha>0$ and assume that $\beta:=\alpha / n<\varepsilon$ for some $n \in \mathbb{N}$. Then $L i_{\beta}$ is universally starlike by assumption, and by Theorem $\mathrm{B}$ we find that the $n$-fold convolution

$$
L i_{\alpha}=L i_{\beta} * L i_{\beta} * \cdots * L i_{\beta}
$$

is universally starlike. Hence the part of Conjecture 1 concerning universal starlikeness has been established. Now let $\alpha \geq 1$. Then by the first part of this proof and Theorem $\mathrm{A}$

$$
\frac{L i_{\alpha-1}(z)}{z}=e^{\int_{[0,1]} \log \frac{1}{1-t z} d \mu(t)}
$$

for some probability measure $\mu$ on $[0,1]$. But then we have

$$
L i_{\alpha}^{\prime}(z)=\frac{L i_{\alpha-1}(z)}{z}=e^{\int_{[0,1]} \log \frac{1}{1-t z} d \mu(t)}=e^{2 \cdot \int_{[0,1]} \log \frac{1}{1-t z} d \mu_{1}(t)},
$$

where $\mu_{1}([0, x]):=\frac{1}{2} \cdot \chi_{(0,1]}(x)+\frac{1}{2} \cdot \mu([0, x]), x \in[0,1]$, is a probability measure. Therefore $L i_{\alpha}$ is universally convex by Theorem A.

The assumption of Lemma 1 is verified in the following assertion.

Lemma 2. For every $\alpha \in(0,1)$ the function $L i_{\alpha}(z) / z$ admits the following representation

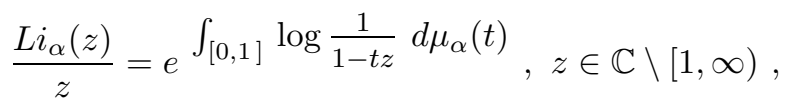

where $\mu_{\alpha}$ is a probability measure on $[0,1]$.

In order to formulate the main result, it is worth recalling once again J. Lewis' remark in [10, concerning the case $\alpha<0$. This remark implies that $L i_{\alpha}$ is not universally convex for $\alpha<0$. Furthermore, it follows from (1.2) that for $z \in \mathbb{D}$

$$
\frac{z \cdot f^{\prime}(z)}{f(z)}=1+z \cdot \int_{0}^{1} \frac{t d \mu(t)}{1-t \cdot z}=1+a_{1} z+\ldots, \quad a_{1}=\int_{0}^{1} t d \mu(t),
$$

and therefore $\left|a_{1}\right| \leq 1$. But since for $\alpha \in \mathbb{R}$

$$
\frac{z \cdot L i_{\alpha}^{\prime}(z)}{L i_{\alpha}(z)}=\frac{L i_{\alpha-1}(z)}{L i_{\alpha}(z)}=1+2^{-\alpha} \cdot z+\ldots,
$$

we have that $L i_{\alpha}$ is not universally starlike for every $\alpha<0$ as well.

Finally, we obtain a positive answer to Conjecture 1.

Theorem 1. Let $\alpha \in \mathbb{R}$. Then the polylogarithm $L i_{\alpha}$ is universally convex respectively universally starlike if and only if $\alpha=0$ or $\alpha \geq 1$, respectively $\alpha \geq 0$. 


\section{Auxiliary Results}

Let $\mathbb{H} \subset \mathbb{C}$ denote the open upper half-plane and $\mathcal{P}$ the class of the so-called Pick functions, i.e.

$$
\mathcal{P}:=\{f \in \operatorname{Hol}(\mathbb{C} \backslash \mathbb{R}) \mid \operatorname{Im} f(z) / \operatorname{Im} z \geq 0 \text { and } f(z)=\overline{f(\bar{z})}, z \in \mathbb{C} \backslash \mathbb{R}\} .
$$

It is well known [2, p.18] that every $f \in \mathcal{P}$ is either a real constant or $\operatorname{Im} f>0$ for all $z \in \mathbb{H}$. Each function in the class $\mathcal{P}$ has a unique representation of the form (see [2, Theorem 1, p.20])

$$
f(z)=a z+b+\int_{-\infty}^{+\infty}\left(\frac{1}{t-z}-\frac{t}{1+t^{2}}\right) d \mu(t)
$$

where $a \geq 0, b$ real, and $\mu$ is a non-negative Borel measure on the real axis for which $\int_{\mathbb{R}}\left(1+t^{2}\right)^{-1} d \mu(t)$ is finite. Conversely, any function of the form (2.1) is in $\mathcal{P}$.

It is also well known (see [2, p.27]) that $\log f \in \mathcal{P}$ for every $f \in \mathcal{P}$ and any function $\varphi$ in the class

$$
\log \mathcal{P}:=\{\log f \mid f \in \mathcal{P}\}
$$

can be represented in the form

$$
\varphi(z)=\sigma+\int_{-\infty}^{+\infty}\left(\frac{1}{t-z}-\frac{t}{1+t^{2}}\right) \rho(t) d t, z \in \mathbb{H},
$$

where $\sigma=\operatorname{Re} \varphi(i) \in \mathbb{R}$ and $\rho$ is a measurable function satisfying $0 \leq \rho(x) \leq 1$, $x \in \mathbb{R}$. Conversely, any function of the form (2.2) is in $\log \mathcal{P}$.

In the sequel for arbitrary function $g \in \bigcup_{p>1} L_{p}(\mathbb{R})$ we use the notation

$$
\widetilde{g}(x):=\frac{1}{\pi} \cdot \int_{0}^{+\infty} \frac{g(x+t)-g(x-t)}{t} d t=\frac{1}{\pi} \cdot \lim _{\varepsilon \downarrow 0} \int_{|t-x|>\varepsilon} \frac{g(t) d t}{t-x},
$$

where $L_{p}(\mathbb{R})$ denotes the real space $L_{p}(\mathbb{R}, d x)$ (see [7, pp.53, 83-85]).

Later on we will use the following known consequence of the M. Riesz theorem (see [8, p.128]).

Theorem D. Let $p>1, g \in L_{p}(\mathbb{R})$, and

$$
G(z)=\frac{1}{\pi} \int_{\mathbb{R}} \frac{g(t) d t}{t-z}, z \in \mathbb{H} .
$$

Then the function $G(z)$ belongs to the $H_{p}$ space for the upper half-plane (see 8 , p. 112]) and for almost every (in short: a.e.) $x \in \mathbb{R}$ (with respect to Lebesgue measure) there exists the (non-tangential) boundary value (see [8, pp.15, 46]) $G(x+i 0)$ $:=\lim _{\mathbb{H} \ni z \rightarrow x} G(z)$ such that

$$
G(x+i 0)=\widetilde{g}(x)+i g(x),
$$

and $\widetilde{g} \in L_{p}(\mathbb{R})$.

The integral representation (1.1) of the function $L i_{\alpha}(z) / z$ with $\alpha \in(0,1)$ implies $L i_{\alpha}(z) / z \in \mathcal{P}$. In fact, writing

$$
v_{\alpha}(x):=\frac{\pi \cdot \chi_{[1,+\infty)}(x)}{\Gamma(1-\alpha) \cdot x \log ^{\alpha} x} \geq 0, x \in \mathbb{R},
$$


then $v_{1-\alpha} \in L_{p}(\mathbb{R}), 1<p<1 /(1-\alpha)$, and we obtain

$$
\frac{L i_{\alpha}(z)}{z}=\frac{1}{\pi} \int_{1}^{+\infty} \frac{v_{1-\alpha}(t) d t}{t-z} .
$$

Thus the representation (2.1) holds for this function with $a=0, d \mu(x)=(1 / \pi)$ $v_{1-\alpha}(x) d x$, and $b=(1 / \pi) \int_{\mathbb{R}}\left[\left(v_{1-\alpha}(t) t d t\right) /\left(1+t^{2}\right)\right]$, and this proves the claim above. Furthermore, Theorem $\mathrm{D}$ applies and proves that $L i_{\alpha}(z) / z$ belongs to the $H_{p}$ spaces of the upper half -plane with $1<p<1 /(1-\alpha)$ and for a.e. $x \in \mathbb{R}$ there exists its boundary value $L i_{\alpha}(x) / x:=L i_{\alpha}(x+i 0) /(x+i 0)$ satisfying

$$
\frac{L i_{\alpha}(x)}{x}=u_{1-\alpha}(x)+i v_{1-\alpha}(x), \quad u_{1-\alpha}:=\widetilde{v}_{1-\alpha} \in L_{p}(\mathbb{R}), \quad 1<p<\frac{1}{1-\alpha} .
$$

We need the following sufficient condition for a function to have a representation as on the right hand side of (1.4).

Lemma 3. Assume that a non-negative function $v$ with $v(x)=0$ for $x<1$ satisfies the following conditions:

(1) $v \in L_{p}(\mathbb{R})$ for some $p>1$,

(2) $\int_{1}^{+\infty} \frac{v(t)}{t} d t=\pi$

(3) for almost all $-\infty<x_{1}<x_{2}<+\infty$ :

$$
\left|\begin{array}{ll}
u\left(x_{1}\right) & u\left(x_{2}\right) \\
v\left(x_{1}\right) & v\left(x_{2}\right)
\end{array}\right| \geq 0
$$

where $u=\widetilde{v}$.

Then there exists a probability measure $\mu$ on $[0,1]$ such that

$$
\frac{1}{\pi} \int_{1}^{+\infty} \frac{v(t) d t}{t-z}=e^{\int_{0}^{1} \log \frac{1}{1-t z} d \mu(t)}, \quad z \in \mathbb{C} \backslash[1, \infty) .
$$

In what follows we shall replace $\alpha$ by $1-\alpha$. We apply Lemma 3 to the function $v=v_{\alpha}$ defined in (2.3). From (1.1) and (2.4) we get

$$
\lim _{z \rightarrow 0} \frac{L i_{1-\alpha}(z)}{z}=1=\frac{1}{\pi} \int_{1}^{+\infty} \frac{v_{\alpha}(t)}{t} d t
$$

and so the second condition of Lemma 3 is satisfied as well as its first one with $p \in(1,1 / \alpha)$ (see (2.5)). It remains to verify the third condition of Lemma 3 with $u=u_{\alpha}:=\widetilde{v}_{\alpha}$.

Note that $v_{\alpha}\left(x_{1}\right)=0$ for $x_{1}<1($ see $(2.3)$ ) and since

$$
u_{\alpha}\left(x_{1}\right)=\widetilde{v}_{\alpha}\left(x_{1}\right)=\frac{1}{\pi} \cdot \int_{1}^{+\infty} \frac{v_{\alpha}(t) d t}{t-x_{1}}>0,
$$

we have

$$
\left|\begin{array}{ll}
u_{\alpha}\left(x_{1}\right) & u_{\alpha}\left(x_{2}\right) \\
v_{\alpha}\left(x_{1}\right) & v_{\alpha}\left(x_{2}\right)
\end{array}\right|=\left|\begin{array}{cc}
u_{\alpha}\left(x_{1}\right) & u_{\alpha}\left(x_{2}\right) \\
0 & v_{\alpha}\left(x_{2}\right)
\end{array}\right|=u_{\alpha}\left(x_{1}\right) \cdot v_{\alpha}\left(x_{2}\right) \geq 0
$$

Thus, to prove condition (3) we have to deal with the cases $1<x_{1}<x_{2}<+\infty$ only. This needs a closer study of the function $u_{\alpha}(x)=\widetilde{v}_{\alpha}(x)$ for $x>1$. 
Lemma 4. Let $\alpha \in(0,1)$ and $v_{\alpha}$ be as in (2.3). Then

$$
\widetilde{v_{\alpha}}(x)=v_{\alpha}(x) \cdot \cot \pi \alpha-\frac{\Gamma(\alpha)}{\pi} \cdot \int_{0}^{+\infty} \frac{K_{\alpha}(t)}{x+t} d t, \quad x>1,
$$

where

$$
K_{\alpha}(t):=\frac{\sin \left[\alpha\left(\frac{\pi}{2}+\arctan \frac{\log t}{\pi}\right)\right]}{t \cdot\left(\log ^{2} t+\pi^{2}\right)^{\alpha / 2}}>0, t>0 .
$$

For $t \in(0,1)$ the expression (2.7) can be rearranged as

$$
K_{\alpha}(t):=\frac{\sin \left[\alpha \arctan \frac{\pi}{\log (1 / t)}\right]}{t \cdot\left(\log ^{2}(1 / t)+\pi^{2}\right)^{\alpha / 2}},
$$

which gives

$$
K_{\alpha}(t)=\frac{\pi \alpha}{t \log ^{1+\alpha}(1 / t)} \cdot(1+o(1)), t \downarrow 0
$$

\section{Proof of Lemma 3}

We write

$h(z):=\frac{1}{\pi} \int_{1}^{+\infty} \frac{v(t) d t}{t-z}=u(z)+i v(z), u(z)=\operatorname{Reh}(z), v(z)=\operatorname{Im} h(z), z \in \mathbb{H}$.

Using the same arguments as for $L i_{\alpha}(z) / z$ in the paragraph preceding the statement of Lemma 3 we find that $h \in \mathcal{P}$ and for almost all $x \in \mathbb{R}$ there exists the boundary value

$$
h(x):=h(x+i 0)=u(x+i 0)+i v(x+i 0)=u(x)+i v(x),
$$

where $u=\widetilde{v} \in L_{p}(\mathbb{R})$.

Let $H:=\log h \in \log \mathcal{P} \subset \mathcal{P}$. Then, with

$$
H(z)=U(z)+i V(z), \quad U(z)=\operatorname{Re} H(z), V(z)=\operatorname{Im} H(z), z \in \mathbb{H},
$$

we have

$$
u(z)=e^{U(z)} \cos V(z), \quad v(z)=e^{U(z)} \sin V(z) .
$$

According to (2.2) there exists a real number $\sigma$ and a measurable function $\rho$ satisfying $0 \leq \rho(x) \leq 1$ a.e. in $\mathbb{R}$ such that

$$
H(z)=\sigma+\int_{-\infty}^{+\infty}\left(\frac{1}{t-z}-\frac{t}{1+t^{2}}\right) \rho(t) d t, z \in \mathbb{H},
$$

and

$$
V(x+i y)=\int_{-\infty}^{+\infty} \frac{y}{(x-t)^{2}+y^{2}} \rho(t) d t
$$

is a non-negative harmonic function in $\mathbb{H}$, bounded by $\pi$. Using the first theorem in [8, p.107] the limit $V(x):=\lim _{\mathbb{H} \ni z \rightarrow \underset{<}{ }} V(z)$ exists almost everywhere on $\mathbb{R}$ and satisfies

$$
V(x)=\pi \cdot \rho(x) \text { a.e. on } \mathbb{R} .
$$


According to Privalov's theorem (see [8, p.62]) we have $u(x)^{2}+v(x)^{2}>0$ a.e. on $\mathbb{R}$ and (3.1) shows that the finite limit $U(x):=\lim _{\mathbb{H} \ni z \rightarrow \underset{<}{ }} U(z)$ also exists a.e. on $\mathbb{R}$, and after taking the limits in (3.1) we get

$$
u(x)=e^{U(x)} \cos \pi \rho(x), \quad v(x)=e^{U(x)} \sin \pi \rho(x) \text { a.e. on } \mathbb{R} .
$$

The third condition of Lemma 3 gives for almost all $-\infty<x_{1}<x_{2}<+\infty$ :

$$
\begin{aligned}
0 & \leq\left|\begin{array}{ll}
u\left(x_{1}\right) & u\left(x_{2}\right) \\
v\left(x_{1}\right) & v\left(x_{2}\right)
\end{array}\right|=\left|\begin{array}{cc}
e^{U\left(x_{1}\right)} \cos \pi \rho\left(x_{1}\right) & e^{U\left(x_{2}\right)} \cos \pi \rho\left(x_{2}\right) \\
e^{U\left(x_{1}\right)} \sin \pi \rho\left(x_{1}\right) & e^{U\left(x_{2}\right)} \sin \pi \rho\left(x_{2}\right)
\end{array}\right| \\
& =e^{U\left(x_{1}\right)+U\left(x_{2}\right)}\left(\cos \pi \rho\left(x_{1}\right) \sin \pi \rho\left(x_{2}\right)-\sin \pi \rho\left(x_{1}\right) \cos \pi \rho\left(x_{2}\right)\right) \\
& =e^{U\left(x_{1}\right)+U\left(x_{2}\right)} \sin \pi\left(\rho\left(x_{2}\right)-\rho\left(x_{1}\right)\right),
\end{aligned}
$$

from which it follows that $\rho\left(x_{2}\right) \geq \rho\left(x_{1}\right)$ for almost all $-\infty<x_{1}<x_{2}<+\infty$. Since (3.2) determines the function $\rho(x)$ a.e. on $\mathbb{R}$ we can consider it as a non-decreasing function, continuous from the left on the whole $\mathbb{R}$ (if necessary redefining it as $\left.\rho(x):=\sup _{y<x} \rho(y), x \in \mathbb{R}\right)$.

Since (3.3) and the second condition of Lemma 3 imply $\rho(x)=0$ a.e. for $x<1$ we have (see e.g. [1, Theorem 11, p.165]) for arbitrary $a>0$ and $z \in \mathbb{H}$

$$
\begin{gathered}
\int_{-\infty}^{+\infty}\left(\frac{1}{t-z}-\frac{t}{1+t^{2}}\right) \rho(t) d t=\int_{1-a}^{+\infty}\left(\frac{1}{t-z}-\frac{t}{1+t^{2}}\right) \rho(t) d t \\
=\int_{1-a}^{+\infty} \rho(t) d\left(\log (t-z)-\frac{1}{2} \log \left(1+t^{2}\right)\right)=\int_{1-a}^{+\infty} \rho(t) d \log \frac{t-z}{\sqrt{1+t^{2}}} \\
=\left.\rho(t) \log \frac{t-z}{\sqrt{1+t^{2}}}\right|_{1-a} ^{+\infty}-\int_{1-a}^{+\infty} \log \frac{t-z}{\sqrt{1+t^{2}}} d \rho(t) \\
=\int_{1-a}^{+\infty} \log \frac{\sqrt{1+t^{2}}}{t-z} d \rho(t)=\int_{1-a}^{+\infty} \log \frac{\sqrt{1+t^{2}}}{t} d \rho(t)+\int_{1-a}^{+\infty} \log \frac{t}{t-z} d \rho(t),
\end{gathered}
$$

and letting $a \downarrow 0$ we get

$$
\int_{-\infty}^{+\infty}\left(\frac{1}{t-z}-\frac{t}{1+t^{2}}\right) \rho(t) d t=\int_{[1,+\infty)} \log \frac{\sqrt{1+t^{2}}}{t} d \rho(t)+\int_{[1,+\infty)} \log \frac{t}{t-z} d \rho(t)
$$

The second integral in (3.4) can be rearranged as follows:

$$
\begin{aligned}
& \int_{[1,+\infty)} \log \frac{t}{t-z} d \rho(t)=-\int_{(0,1]} \log \frac{1}{1-t z} d \rho(1 / t) \\
= & \int_{(0,1]} \log \frac{1}{1-t z} d(1-\rho(1 / t))=\int_{[0,1]} \log \frac{1}{1-t z} d \nu(t),
\end{aligned}
$$

where $\nu$ is the non-decreasing function on $\mathbb{R}$, continuous from the right, defined as

$$
\nu(t):=\left\{\begin{array}{lc}
0, & t<0 \\
1-\rho(+\infty), & t=0 \\
1-\rho(1 / t), & 0<t<1 \\
1, & t \geq 1
\end{array}\right.
$$

Here $\nu(0-0)=0, \nu(0)=\nu(0+0)=1-\rho(+\infty), \nu(1-0)=1-\rho(1+0), \nu(1)=$ $\nu(1+0)=1$. Thus, the function $\nu$ defines (see e.g. [9, Proposition 3.9, p.145]) 
a probability measure on $[0,1]$ and

$$
h(z)=e^{H(z)}=e^{C} \cdot e^{\int_{[0,1]} \log \frac{1}{1-t z} d \nu(t)}, \quad C:=\sigma+\int_{[1,+\infty)} \log \frac{\sqrt{1+t^{2}}}{t} d \rho(t) .
$$

For $z=0$ we get

$$
e^{C}=h(0)=\frac{1}{\pi} \int_{1}^{+\infty} \frac{v(t) d t}{t}=1
$$

and so

$$
h(z)=e^{\int_{[0,1]} \log \frac{1}{1-t z} d \nu(t)},
$$

the claim.

\section{Proof of Lemma 4}

For a given $\delta>0$ we introduce the function

$$
T_{\delta}(z):=\frac{e^{i \pi \alpha}}{(z+i \delta) \cdot \log ^{\alpha} z}, \quad z \in \mathbb{H} .
$$

For $z=r e^{i \varphi}, \quad 0<\varphi<\pi$ we have $z \in \mathbb{H}$ and $z+i \delta \in \mathbb{H}$. Straightforward calculation then yields

$$
T_{\delta}(z)=\frac{\exp \left[i \frac{\pi(\alpha-1)}{2}+i \alpha \arctan \frac{\log r}{\varphi}+i \arctan \frac{r \cos \varphi}{\delta+r \sin \varphi}\right]}{\left((\log r)^{2}+\varphi^{2}\right)^{\alpha / 2}\left(r^{2}+2 r \delta \sin \varphi+\delta^{2}\right)^{\frac{1}{2}}}
$$

and

$$
T_{\delta}(x+i 0)=\left\{\begin{array}{cc}
\frac{\exp \left[i \pi \alpha-i \frac{\pi}{2}+i \arctan \frac{x}{\delta}\right]}{\left(x^{2}+\delta^{2}\right)^{\frac{1}{2}} \cdot \log ^{\alpha} x}, & x>1, \\
\frac{\exp \left[-i \frac{\pi}{2}+i \arctan \frac{x}{\delta}\right]}{\left(x^{2}+\delta^{2}\right)^{\frac{1}{2}} \cdot \log ^{\alpha} \frac{1}{x}}, & x \in(0,1), \\
\frac{\exp \left[i \frac{\pi(\alpha-1)}{2}-i \arctan \frac{|x|}{\delta}+i \alpha \arctan \frac{\log |x|}{\pi}\right]}{\left(\log ^{2}|x|+\pi^{2}\right)^{\alpha / 2}\left(x^{2}+\delta^{2}\right)^{\frac{1}{2}}}, & x<0 .
\end{array}\right.
$$

It is easy to see that $T_{\delta}(x+i 0)$ has a singularity at $x=1$, proportional to $|x-1|^{-\alpha}$, and approaches zero like $|x|^{-1}$ when $|x| \rightarrow+\infty$. Therefore $\left|T_{\delta}(x+i 0)\right| \in L_{p}(\mathbb{R})$ for any $p \in(1,1 / \alpha)$. According to Theorem $\mathrm{D}$ we have

$$
T_{\delta}(z)=\frac{1}{\pi} \int_{\mathbb{R}} \frac{\operatorname{Im} T_{\delta}(t+i 0) d t}{t-z}, z \in \mathbb{H} .
$$

Now let

$$
R_{\delta}(z):=\frac{1}{\pi} \int_{-\infty}^{1} \frac{\operatorname{Im} T_{\delta}(t+i 0) d t}{t-z}, z \in \mathbb{H}
$$

then

$$
T_{\delta}(z)-R_{\delta}(z)=\frac{1}{\pi} \int_{1}^{+\infty} \frac{\operatorname{Im} T_{\delta}(t+i 0) d t}{t-z}, z \in \mathbb{H}
$$


and Theorem $\mathrm{D}$ establishes the existence of the boundary values of $R_{\delta}(z)$ and $T_{\delta}-R_{\delta}$. The latter, by (4.1), leads to

$$
\begin{gathered}
w_{\delta}(x):=\operatorname{Im}\left(T_{\delta}-R_{\delta}\right)(x+i 0)=\operatorname{Im} \frac{e^{i \pi \alpha-i \frac{\pi}{2}+i \arctan \frac{x}{\delta}}}{\left(x^{2}+\delta^{2}\right)^{\frac{1}{2}} \cdot \log ^{\alpha} x} \cdot \chi_{[1,+\infty)}(x) \\
=-\frac{\cos \left(\pi \alpha+\arctan \frac{x}{\delta}\right)}{\left(x^{2}+\delta^{2}\right)^{\frac{1}{2}} \cdot \log ^{\alpha} x} \cdot \chi_{[1,+\infty)}(x), x \in \mathbb{R} .
\end{gathered}
$$

This, together with (4.1), now gives for a.e. $x>1$

$$
\begin{aligned}
\widetilde{w_{\delta}}(x) & =\operatorname{Re}\left(T_{\delta}-R_{\delta}\right)(x+i 0) \\
& =\frac{\sin \left(\pi \alpha+\arctan \frac{x}{\delta}\right)}{\left(x^{2}+\delta^{2}\right)^{\frac{1}{2}} \cdot \log ^{\alpha} x}-\frac{1}{\pi} \cdot \operatorname{Re} \lim _{y \downarrow 0} \int_{-\infty}^{1} \frac{\operatorname{Im} T_{\delta}(t+i 0) d t}{t-x-i y},
\end{aligned}
$$

but

$$
\begin{gathered}
-\operatorname{Re} \lim _{y \downarrow 0} \int_{-\infty}^{1} \frac{\operatorname{Im} T_{\delta}(t+i 0) d t}{t-x-i y}=\int_{-\infty}^{0} \frac{\operatorname{Im} T_{\delta}(t+i 0) d t}{x-t}+\int_{0}^{1} \frac{\operatorname{Im} T_{\delta}(t+i 0) d t}{x-t} \\
\stackrel{\text { 44.1) }}{=} \int_{0}^{\infty} \frac{\operatorname{Im} T_{\delta}(-t+i 0) d t}{x+t}-\int_{0}^{1} \frac{\cos \arctan \frac{t}{\delta} d t}{(x-t)\left(t^{2}+\delta^{2}\right)^{\frac{1}{2}} \cdot \log ^{\alpha} \frac{1}{t}} \\
=-\int_{0}^{\infty} \frac{\cos \left(\frac{\pi \alpha}{2}-\arctan \frac{t}{\delta}+\alpha \arctan \frac{\log t}{\pi}\right) d t}{(x+t)\left(\log ^{2} t+\pi^{2}\right)^{\alpha / 2}\left(t^{2}+\delta^{2}\right)^{\frac{1}{2}}}-\int_{0}^{1} \frac{\delta d t}{(x-t)\left(t^{2}+\delta^{2}\right) \cdot \log ^{\alpha} \frac{1}{t}},
\end{gathered}
$$

from which we get

$$
\begin{gathered}
\widetilde{w_{\delta}}(x)=\frac{\sin \left(\pi \alpha+\arctan \frac{x}{\delta}\right)}{\left(x^{2}+\delta^{2}\right)^{\frac{1}{2}} \cdot \log ^{\alpha} x}-\frac{1}{\pi} \cdot \int_{0}^{1} \frac{\delta d t}{(x-t)\left(t^{2}+\delta^{2}\right) \cdot \log ^{\alpha} \frac{1}{t}} \\
-\frac{1}{\pi} \cdot \int_{0}^{\infty} \frac{\cos \left(\frac{\pi \alpha}{2}-\arctan \frac{t}{\delta}+\alpha \arctan \frac{\log t}{\pi}\right) d t}{(x+t)\left(\log ^{2} t+\pi^{2}\right)^{\alpha / 2}\left(t^{2}+\delta^{2}\right)^{\frac{1}{2}}}, \quad x>1,
\end{gathered}
$$

using the continuity of $\widetilde{w_{\delta}}(x)$ at every point $x>1$ (see [8, p.18]).

Next we show that the functions $w_{\delta}(x), \delta>0$, which belong to $L_{p}(\mathbb{R})$ for any $p \in(1,1 / \alpha)$, converge in each of these spaces as follows:

$$
\lim _{\delta \downarrow 0} w_{\delta}(x)=\frac{\sin \pi \alpha}{x \cdot \log ^{\alpha} x} \cdot \chi_{[1,+\infty)}(x)=\frac{\Gamma(1-\alpha) \sin \pi \alpha}{\pi} \cdot v_{\alpha}(x)=: w_{0}(x) .
$$

Actually, for $x>1$

$$
w_{\delta}(x)=\frac{x}{\left(x^{2}+\delta^{2}\right) \cdot \log ^{\alpha} x} \cdot \sin \pi \alpha-\frac{\delta}{\left(x^{2}+\delta^{2}\right) \cdot \log ^{\alpha} x} \cdot \cos \pi \alpha,
$$

and then

$$
w_{0}(x)-w_{\delta}(x)=\frac{\delta}{\left(x^{2}+\delta^{2}\right) \cdot \log ^{\alpha} x} \cdot \cos \pi \alpha+\frac{\delta^{2}}{x \cdot\left(x^{2}+\delta^{2}\right) \cdot \log ^{\alpha} x} \cdot \sin \pi \alpha .
$$


It is evident that the $L_{p}$-norm over the interval $[1,+\infty)$ of each of the two terms of the function in (4.4) tends to zero for $\delta \downarrow 0$, provided $p \in(1,1 / \alpha)$. Therefore, in view of continuity of the Hilbert transformation in these spaces ( M. Riesz' theorem, see [8, p.127]) we have that for a.e. $x>1$

$$
\lim _{\delta \downarrow 0} \widetilde{w_{\delta}}(x)=\widetilde{w_{0}}(x) \equiv \widetilde{v_{\alpha}}(x) \cdot \frac{\Gamma(1-\alpha) \sin \pi \alpha}{\pi} .
$$

Hence, in order to obtain a suitable integral representation for $\widetilde{v_{\alpha}}(x)$ it remains to perform the passage $\delta \downarrow 0$ in (4.2) suitably.

It follows from (4.2) and (4.5) that for a.e. $x>1$

$$
\widetilde{w_{0}}(x)=\frac{\cos \pi \alpha}{x \cdot \log ^{\alpha} x}-\lim _{\delta \downarrow 0} \frac{1}{\pi} \cdot \int_{0}^{1} \frac{A_{\delta}(t)}{x-t} d t-\lim _{\delta \downarrow 0} \frac{1}{\pi} \cdot \int_{0}^{\infty} \frac{B_{\delta}(t)}{x+t} d t,
$$

where

$$
\begin{aligned}
A_{\delta}(t) & :=\frac{\delta}{\left(t^{2}+\delta^{2}\right) \cdot \log ^{\alpha} \frac{1}{t}} \\
B_{\delta}(t) & :=B_{\delta}^{0}(t)+B_{\delta}^{1}(t) \\
B_{\delta}^{0}(t) & :=\frac{\delta \cos \alpha\left[\frac{\pi}{2}+\arctan \frac{\log t}{\pi}\right]}{\left(\log ^{2} t+\pi^{2}\right)^{\alpha / 2}\left(t^{2}+\delta^{2}\right)}, \\
B_{\delta}^{1}(t) & :=\frac{t \sin \alpha\left[\frac{\pi}{2}+\arctan \frac{\log t}{\pi}\right]}{\left(\log ^{2} t+\pi^{2}\right)^{\alpha / 2}\left(t^{2}+\delta^{2}\right)} .
\end{aligned}
$$

Let $q:=\sqrt[4]{\delta}$. We assume that $\delta<1$, so that

$$
\int_{0}^{1}\left|\frac{A_{\delta}(t)}{x-t}\right| d t \leq \frac{1}{x-1} \cdot \int_{0}^{1} \frac{\delta}{\left(t^{2}+\delta^{2}\right) \cdot \log ^{\alpha} \frac{1}{t}} d t=\frac{1}{x-1} \cdot\left(\int_{0}^{q}+\int_{q}^{1}\right),
$$

where

$$
\int_{0}^{q} \leq \frac{1}{\log ^{\alpha} \frac{1}{q}} \int_{0}^{q} \frac{\delta}{\left(t^{2}+\delta^{2}\right)} d t \leq \frac{4^{\alpha}}{\log ^{\alpha} \frac{1}{\delta}} \int_{0}^{\infty} \frac{1}{\left(t^{2}+1\right)} d t
$$

and

$$
\int_{q}^{1} \leq \sqrt{\delta} \cdot \int_{q}^{1} \frac{1}{\log ^{\alpha} \frac{1}{t}} d t \leq \sqrt{\delta} \cdot \int_{0}^{1} \frac{1}{\log ^{\alpha} \frac{1}{t}} d t=\sqrt{\delta} \cdot \Gamma(1-\alpha)
$$

Hence

$$
\lim _{\delta \downarrow 0} \int_{0}^{1} \frac{A_{\delta}(t)}{x-t} d t=0 \quad, \quad x>1
$$

From (4.7) we deduce

$$
\left|\int_{0}^{\infty} \frac{B_{\delta}^{0}(t)}{x+t} d t\right| \leq \int_{0}^{\infty} \frac{\delta d t}{(x+t) \cdot\left(t^{2}+\delta^{2}\right)\left(\log ^{2} t+\pi^{2}\right)^{\alpha / 2}}=\int_{0}^{q}+\int_{q}^{1 / q}+\int_{1 / q}^{\infty},
$$

where

$$
\int_{0}^{q} \leq \frac{1}{x} \cdot \frac{1}{\left(\log ^{2} q+\pi^{2}\right)^{\alpha / 2}} \int_{0}^{q} \frac{\delta d t}{t^{2}+\delta^{2}} \leq \frac{4^{\alpha}}{x \cdot \log ^{\alpha} \frac{1}{\delta}} \int_{0}^{\infty} \frac{\delta d t}{t^{2}+\delta^{2}}=\frac{4^{\alpha} \pi}{2 x \cdot \log ^{\alpha} \frac{1}{\delta}},
$$




$$
\begin{aligned}
\int_{q}^{1 / q} & \leq \frac{\sqrt{\delta}}{\pi^{\alpha}} \int_{q}^{1 / q} \frac{d t}{x+t} \leq \frac{\sqrt{\delta}}{\pi^{\alpha}} \int_{q}^{1 / q} \frac{d t}{1+t}=\frac{\sqrt{\delta}}{\pi^{\alpha}} \log \frac{1+1 / q}{1+q} \leq \frac{\sqrt{\delta}}{\pi^{\alpha}} \log \frac{1}{\sqrt{\delta}} \\
\int_{1 / q}^{\infty} & \leq \frac{1}{\pi^{\alpha}\left(x+\frac{1}{q}\right)} \int_{1 / q}^{\infty} \frac{\delta d t}{\left(t^{2}+\delta^{2}\right)} \leq \frac{q}{\pi^{\alpha}} \int_{0}^{\infty} \frac{\delta d t}{\left(t^{2}+\delta^{2}\right)}=\frac{\pi^{1-\alpha}}{2} \cdot \sqrt[4]{\delta}
\end{aligned}
$$

so that

$$
\lim _{\delta \downarrow 0} \int_{0}^{\infty} \frac{B_{\delta}^{0}(t)}{x+t} d t=0 \quad, \quad x>1
$$

In order to evaluate

$$
\lim _{\delta \downarrow 0} \frac{1}{\pi} \cdot \int_{0}^{\infty} \frac{B_{\delta}^{1}(t)}{x+t} d t
$$

using Lebesgue's dominated convergence theorem [11, p.26], we note that

$$
B_{0}^{1}(t):=\lim _{\delta \downarrow 0} B_{\delta}^{1}(t)=\frac{\sin \left[\alpha\left(\frac{\pi}{2}+\arctan \frac{\log t}{\pi}\right)\right]}{t \cdot\left(\log ^{2} t+\pi^{2}\right)^{\alpha / 2}},
$$

and

$$
\left|\frac{B_{\delta}^{1}(t)}{x+t}\right|=\left|\frac{t^{2} \sin \left[\alpha\left(\frac{\pi}{2}+\arctan \frac{\log t}{\pi}\right)\right]}{\left(t^{2}+\delta^{2}\right) \cdot t \cdot(x+t) \cdot\left(\log ^{2} t+\pi^{2}\right)^{\alpha / 2}}\right| \leq \frac{\left|B_{0}^{1}(t)\right|}{x+t}
$$

hold for $t>0$. The function on the right hand side of (4.10) is obviously integrable in $t$ at infinity. We need to show that this is also true at $t=0$.

To do this we rearrange the argument of the sine function in (4.9) for $t \in(0,1)$,

$$
\frac{\pi}{2}+\arctan \frac{\log t}{\pi}=\frac{\pi}{2}-\arctan \frac{\log (1 / t)}{\pi}=\arctan \frac{\pi}{\log (1 / t)},
$$

and for $t \downarrow 0$,

$$
\sin \left[\alpha\left(\frac{\pi}{2}+\arctan \frac{\log t}{\pi}\right)\right]=\sin \left[\alpha\left(\frac{\pi}{\log (1 / t)}+O\left(\frac{1}{\log ^{2}(1 / t)}\right)\right)\right] .
$$

This implies

$$
B_{0}^{1}(t)=\frac{\pi \alpha}{t \cdot \log ^{1+\alpha}(1 / t)} \cdot(1+o(1)), t \downarrow 0,
$$

and so this function is integrable in $t$ on any interval $[0, a], a>0$. This, together with (4.7), (4.8), (4.9) and the continuity of $\widetilde{w_{0}}(x)$ at every point $x>1$ (see [8, p.18]), shows that taking the limits in (4.6) yields

$$
\widetilde{w_{0}}(x)=\cot (\pi \alpha) \cdot w_{0}(x)-\frac{1}{\pi} \cdot \int_{0}^{\infty} \frac{\sin \left[\alpha\left(\frac{\pi}{2}+\arctan \frac{\log t}{\pi}\right)\right]}{(x+t) \cdot t \cdot\left(\log ^{2} t+\pi^{2}\right)^{\alpha / 2}} d t, \quad x>1 .
$$

Next we recall (4.3) to obtain (essentially) the expression in (2.6). Equalities (2.8) and (2.9) follow from (4.11) and (4.12), respectively. The proof is complete. 


\section{Proof of Lemma 2}

For the proof of Lemma 2 it suffices to establish that for almost all $1<x_{1}<$ $x_{2}<+\infty$ we have

$$
\left|\begin{array}{ll}
u_{\alpha}\left(x_{1}\right) & u_{\alpha}\left(x_{2}\right) \\
v_{\alpha}\left(x_{1}\right) & v_{\alpha}\left(x_{2}\right)
\end{array}\right| \geq 0
$$

where, according to Lemma 4 , and for $x>1$ we have to use

$$
\begin{aligned}
v_{\alpha}(x) & :=\frac{\pi}{\Gamma(1-\alpha)} \cdot \frac{1}{x \log ^{\alpha} x} \\
v_{\alpha}^{\prime}(x) & =-v_{\alpha}(x) \cdot\left(\frac{1}{x}+\frac{\alpha}{x \log x}\right), \\
u_{\alpha}(x) & =v_{\alpha}(x) \cdot \cot \pi \alpha-\frac{\Gamma(\alpha)}{\pi} \cdot \int_{0}^{+\infty} \frac{K_{\alpha}(t)}{x+t} d t \\
u_{\alpha}^{\prime}(x) & =v_{\alpha}^{\prime}(x) \cdot \cot \pi \alpha+\frac{\Gamma(\alpha)}{\pi} \cdot \int_{0}^{+\infty} \frac{K_{\alpha}(t)}{(x+t)^{2}} d t .
\end{aligned}
$$

Direct calculation yields

$$
\left|\begin{array}{ll}
u_{\alpha}^{\prime}(x) & u_{\alpha}(x) \\
v_{\alpha}^{\prime}(x) & v_{\alpha}(x)
\end{array}\right|=-v_{\alpha}(x) \cdot\left(\int_{0}^{+\infty} \frac{\Gamma(1+\alpha) \cdot K_{\alpha}(t)}{\pi x \cdot(x+t) \cdot \log x} d t+\int_{0}^{+\infty} \frac{\Gamma(\alpha) \cdot t \cdot K_{\alpha}(t)}{\pi x \cdot(x+t)^{2}} d t\right) .
$$

Therefore, using the fact that $K_{\alpha}(t)>0$ for $t \in(0, \infty)$ and $\alpha \in(0,1)$, we find

$$
\begin{gathered}
v_{\alpha}(x)^{2} \cdot \frac{d}{d x} \frac{u_{\alpha}(x)}{v_{\alpha}(x)}=u_{\alpha}^{\prime}(x) v_{\alpha}(x)-u_{\alpha}(x) v_{\alpha}^{\prime}(x) \\
=-\frac{\pi}{\Gamma(1-\alpha)} \cdot \frac{1}{x \log ^{\alpha} x}\left(\int_{0}^{+\infty} \frac{\Gamma(1+\alpha) \cdot K_{\alpha}(t)}{\pi x \cdot(x+t) \cdot \log x} d t+\int_{0}^{+\infty} \frac{\Gamma(\alpha) \cdot t \cdot K_{\alpha}(t)}{\pi x \cdot(x+t)^{2}} d t\right)<0 .
\end{gathered}
$$

This means that for $x>1$ the function $\frac{u_{\alpha}(x)}{v_{\alpha}(x)}$ strictly decreases and thus, for arbitrary $1<x_{2}<x_{2}<+\infty$ :

$$
\frac{u_{\alpha}\left(x_{2}\right)}{v_{\alpha}\left(x_{2}\right)}<\frac{u_{\alpha}\left(x_{1}\right)}{v_{\alpha}\left(x_{1}\right)}
$$

i.e.

$$
0<u_{\alpha}\left(x_{1}\right) v_{\alpha}\left(x_{2}\right)-u_{\alpha}\left(x_{2}\right) v_{\alpha}\left(x_{1}\right)=\left|\begin{array}{ll}
u_{\alpha}\left(x_{1}\right) & u_{\alpha}\left(x_{2}\right) \\
v_{\alpha}\left(x_{1}\right) & v_{\alpha}\left(x_{2}\right)
\end{array}\right|,
$$

which proves the claim of Lemma 2 ,

\section{REFERENCES}

[1] Gearoid de Barra, Measure theory and integration, Ellis Horwood Ltd., Chichester, 1981. Ellis Horwood Series in Mathematics and its Applications. MR637464 (84h:28001)

[2] William F. Donoghue Jr., Monotone matrix functions and analytic continuation, SpringerVerlag, New York, 1974. Die Grundlehren der mathematischen Wissenschaften, Band 207. MR.0486556 (58 \#6279)

[3] Peter L. Duren, Univalent functions, Grundlehren der Mathematischen Wissenschaften [Fundamental Principles of Mathematical Sciences], vol. 259, Springer-Verlag, New York, 1983. MR708494 (85j:30034) 
[4] Arthur Erdélyi, Wilhelm Magnus, Fritz Oberhettinger, and Francesco G. Tricomi, Higher transcendental functions. Vol. I, Robert E. Krieger Publishing Co. Inc., Melbourne, Fla., 1981. Based on notes left by Harry Bateman; With a preface by Mina Rees; With a foreword by E. C. Watson; Reprint of the 1953 original. MR698779 (84h:33001a)

[5] Alexander B. Goncharov, Polylogarithms in arithmetic and geometry, 2 (Zürich, 1994), Birkhäuser, Basel, 1995, pp. 374-387. MR1403938 (97h:19010)

[6] Pedro Jodrá, On a connection between the polylogarithm function and the Bass diffusion model, Proc. R. Soc. Lond. Ser. A Math. Phys. Eng. Sci. 464 (2008), no. 2099, 3081-3088, DOI 10.1098/rspa.2008.0196. MR2439315 (2009h:33004)

[7] Frederick W. King, Hilbert transforms. Vol. 1, Encyclopedia of Mathematics and its Applications, vol. 124, Cambridge University Press, Cambridge, 2009. MR2542214 (2011c:42003)

[8] Paul Koosis, Introduction to $H_{p}$ spaces, 2nd ed., Cambridge Tracts in Mathematics, vol. 115, Cambridge University Press, Cambridge, 1998. With two appendices by V. P. Havin [Viktor Petrovich Khavin]. MR 1669574 (2000b:30052)

[9] A. Mukherjea and K. Pothoven, Real and functional analysis, Plenum Press, New York, 1978. Mathematical Concepts and Methods in Science and Engineering, Vol. 6. MR0492145 $(58$ \#11294)

[10] John L. Lewis, Convexity of a certain series, J. London Math. Soc. (2) 27 (1983), no. 3, 435-446, DOI 10.1112/jlms/s2-27.3.435. MR697137 (84d:30021)

[11] Walter Rudin, Real and complex analysis, 2nd ed., McGraw-Hill Book Co., New York, 1974. McGraw-Hill Series in Higher Mathematics. MR0344043 (49 \#8783)

[12] St. Ruscheweyh and T. Sheil-Small, Hadamard products of Schlicht functions and the PólyaSchoenberg conjecture, Comment. Math. Helv. 48 (1973), 119-135. MR0328051 (48 \#6393)

[13] Stephan Ruscheweyh and Luis Salinas, Universally prestarlike functions as convolution multipliers, Math. Z. 263 (2009), no. 3, 607-617, DOI 10.1007/s00209-008-0433-3. MR2545859 (2010h:30028)

[14] Stephan Ruscheweyh, Luis Salinas, and Toshiyuki Sugawa, Completely monotone sequences and universally prestarlike functions, Israel J. Math. 171 (2009), 285-304, DOI 10.1007/s11856-009-0050-9. MR2520111(2010e:30012)

Institute of Mathematics, National Academy of Sciences of Ukraine, Kyiv 01601, UKRAINE

E-mail address: andrew@bakan.kiev.ua

Institut für Mathematik, Universität Würzburg, 97074 Würzburg, Germany

E-mail address: ruscheweyh@mathematik.uni-wuerzburg.de

Departamento de Informática, UTFSm, Valparaíso, Chile

E-mail address: luis.salinas@usm.cl 